\title{
Editorial
}

\section{Precision Medicine in Neurocritical Care-Finding Opportunities and Overcoming the Obstacles}

\author{
Zulfiqar Ali ${ }^{1}$ Hemanshu Prabhakar ${ }^{2}$ \\ ${ }^{1}$ Division of Neuroanesthesiology and Neurocritical Care, SKIMS, \\ Soura, Srinagar, India \\ ${ }^{2}$ Department of Neuroanesthesiology and Critical Care, AlIMS, \\ New Delhi, India
}

Indian J Neurosurg 2019;8:149-150

Advances in neurocritical care over the last decade have resulted in significant improvement in patient outcomes. With rapid advances in the technology, there has been an improvement in neurologic monitoring, resulting in improved outcomes in patients with severe traumatic brain injury, intracerebral hemorrhage, stroke, and aneurysmal subarachnoid hemorrhage. Precision medicine with the availability of accurate electronic medical data coupled with precise laboratory tests, easy availability and access of imaging modalities may help in unravelling the complex pathophysiological processes in neurosurgical patients facilitating the achievement of "Personalized, Preventive, Predictive, and Participatory" components (four Ps) of precision medicine.

The initial precision medicine evolved from medical oncology, where the use of genetic biomarkers transformed the therapeutic interventions. ${ }^{1}$ The growth in molecular oncology created a better understanding of malignancies and identified exploitable targets which influenced the disease outcomes. However, the application of precision medicine in neurointensive care, fraught with complex multitude of medical issues, has been very slow. The complex nature of illness influenced by multiple host factors, with a lack of appropriate clinical trials with a class I or class II evidence, may be responsible for impediment to the application of precision medicine in a neurocritical care setting.

A closed-head injury patient may be associated with fracture of a long bone or a hemoperitoneum where precise treatment of one pathology may have no effect or may adversely affect the other medical issue. Should we give a massive fluid transfusion to treat hypotension in a closed head injury with hemoperitoneum or will it adversely affect the intracranial hemodynamics? Precision medicine may help in answering such questions in the coming years. A neurosurgical ICU which houses patients with the greatest severity of illness may offer a great opportunity, for improving patient survival and quality of life due to application of the precision medicine.
The major impediment to precision medicine includes a lack of the clarity and marked variability in working definitions. Unfortunately we do not even have a definition of the 'critically ill' patient in a neurosurgical unit or defining critical events in neurointensive care unit as a Cardiac arrest in intensive care unit! ${ }^{2,3}$ This marked variability makes it difficult to study a disease or to target treatment in a critically ill patient. Adding to the pandemonium, there is a dilemma among the clinicians working in a neurocritical care unit for various point-of-care monitoring systems. There is a lack of clarity on point of care testing for glycemic control on blood glucose monitoring devices approved for use in critically ill patients, with significant differences in different laboratory platforms using paired specimens. ${ }^{4}$ Lack of standardization with significant discordance of values and biological variability of the biomarkers exists in laboratory testing methodology to assess the perfusion, coagulation, renal function, hepatic function and cardiac injury. Lactate as a marker of perfusion in critical care, is not standardized, with different laboratory platforms with a marked discordance as values increase to $4 \mathrm{mmol} / \mathrm{L}$ and beyond. ${ }^{5}$ There are significant differences between point of care testing and laboratory platforms in assessing the coagulation profile, with multiple assays using rabbit thromboplastin and recombinant human thromboplastin. ${ }^{6}$ Despite standardization of measurement techniques for creatinine by standardized reference method using isotope dilution mass spectrometry, there is still a significant biological variability of the biomarker causing a substantial discordance between values of paired samples using different platforms. ${ }^{7}$ Biologically released troponin can be monomeric, dimeric, or trimeric with differing epitopes detected by each immunoassay. Hence a lot of variability can be observed between troponin I assays and $\mathrm{T}$ assays using different laboratory platforms. ${ }^{8,9}$ Hence a profound knowledge of how samples are analyzed and what they mean is essential if precision medicine is to be 'precise'.

Due to the multifaceted nature of injury involving multiple organ systems in neurosurgical patients, both
Address for correspondence Zulfiqar Ali, MD, DM, Division of Neuroanesthesiology and Neurocritical Care, SKIMS, Soura, Srinagar 190011, India (e-mail: zulfiqaraliiii@yahoo.com).
DOI https://doi.org/ $10.1055 / \mathrm{s}-0039-3401439$ ISSN 2277-954X.
C2019 Neurological Surgeons' Society of India
License terms

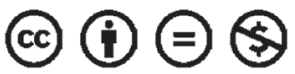


prognostic precision and predictive precision may improve outcome in neurocritical care. Prognostic patient selection involves the selection of patients with a greater chance of a disease-related event, such as mortality due to sepsis, ${ }^{10}$ whereas predictive selection involves selection of patients more likely to respond to an intervention based on biological mechanisms associated with a disease, as the utility of pharmacogenomics, in targeted use of antibiotics in sepsis. ${ }^{11}$ Similarly the use of biomarkers may predict the responsiveness and outcome in ARDS to the application of PEEP ${ }^{12}$ and fluid management. ${ }^{13}$

The wide use of antiepileptics in neurocritical care may influence the cytochrome P 450 metabolism of narcotics and antimicrobials affecting the plasma concentration of these drugs. Pharmacogenomics, evaluating the influence of the patient genomic makeup on pharmacokinetics and pharmacodynamics, will play a role in the future ICU precision medicine practice, to optimize the doses of antimicrobials and narcotics.

Current electronic health record systems are full of inconsistent and inaccurate data due to human limitations. Consistency in data acquisition, storage and reporting will require an unparalleled collaboration that has never been possible in the field of medicine till date. New data management and analytic techniques using machine learning and artificial intelligence will help to integrate the data from patients to aid in the development of algorithms in future, based on precision based medicine. This data obtained from subsets of neurosurgical populations may help to develop valid and clinically meaningful data for treatment of patients with head injury, intracranial tumors, spinal cord injury, subarachnoid hemorrhage, and other subsets of population in intensive care unit. This may lead to development of registry-based randomized controlled trial (that will use data collected from registry data; to identify appropriate targets for trial enrolment) ${ }^{14}$ and the platform trial. ${ }^{15}$ (which uses response-adaptive randomization to test multiple treatments in a pre-specified patients using Bayesian analysis.)

Precision medicine development in neurocritical care is in infancy. It is in need of cohort identification in subsets of patient population admitted to the neurointensive care unit. Informed groups consisting of neuroanesthesiologists, neurointensivists, neurosurgeons, neuropathologists, neurologists, biostatisticians, neuro epidemiologists, neuroendocrinologists and neuro pharmacologists need to sit together to understand, gather and analyze data on various neurological disorders. This will help in an accurate identification of the key steps needed to identify subpopulations for prognostic markers.

Though, the vision for precision medicine in the neuro critical care is now visible on the horizon, however, the road is not smooth with a path full of barricades and a disorganised construction team. Success will depend on optimal strategic resource utilization, collaboration between various subspecialties, use of newer technologies and introduction of newer clinical trial designs.

Conflict of Interest

None declared.

\section{References}

1 Collins FS, Varmus H. A new initiative on precision medicine. N Engl J Med 2015;372(9):793-795

2 Anthi A, Tzelepis GE, Alivizatos P, Michalis A, Palatianos GM, Geroulanos S. Unexpected cardiac arrest after cardiac surgery: incidence, predisposing causes, and outcome of open chest cardiopulmonary resuscitation. Chest 1998;113(1):15-19

3 Efendijev I, Raj R, Reinikainen M, Hoppu S, Skrifvars MB. Temporal trends in cardiac arrest incidence and outcome in Finnish intensive care units from 2003 to 2013. Intensive Care Med 2014;40(12):1853-1861

4 Kost GJ, Tran NK, Louie RF, Gentile NL, Abad VJ. Assessing the performance of handheld glucose testing for critical care. Diabetes Technol Ther 2008;10(6):445-451

5 Karon BS, Scott R, Burritt MF, Santrach PJ. Comparison of lactate values between point-of-care and central laboratory analyzers. Am J Clin Pathol 2007;128(1):168-171

6 Poller L. International Normalized Ratios (INR): the first 20 years. J Thromb Haemost 2004;2(6):849-860

7 Piéroni L, Bargnoux AS, Cristol JP, Cavalier E, Delanaye P. Did creatinine standardization give benefits to the evaluation of glomerular filtration rate. EJIFCC 2017;28(4):251-257

8 Christenson RH, Bunk DM, Schimmel H, Tate JR; IFCC Working Group on Standardization of Troponin I. Point: Put simply, standardization of cardiac troponin I is complicated. Clin Chem 2012;58(1):165-168

9 Gaze DC, Collinson PO. Multiple molecular forms of circulating cardiac troponin: analytical and clinical significance. Ann Clin Biochem 2008;45(Pt 4):349-355

10 Langley RJ, Tsalik EL, van Velkinburgh JC, et al. An integrated clinico-metabolomic model improves prediction of death in sepsis. Sci Transl Med 2013;5(195):195ra95

11 Aung AK, Haas DW, Hulgan T, Phillips EJ. Pharmacogenomics of antimicrobial agents. Pharmacogenomics 2014;15(15):1903-1930

12 Calfee CS, Delucchi K, Parsons PE, Thompson BT, Ware LB, Matthay MA; NHLBI ARDS Network. Subphenotypes in acute respiratory distress syndrome: latent class analysis of data from two randomised controlled trials. Lancet Respir Med 2014;2(8):611-620

13 Famous KR, Delucchi K, Ware LB, et al; ARDS Network. Acute Respiratory Distress Syndrome Subphenotypes Respond Differently to Randomized Fluid Management Strategy. Am J Respir Crit Care Med 2017;195(3):331-338

14 Lauer MS. D'Agostino RB Sr. The randomized registry trial-the next disruptive technology in clinical research? N Engl J Med 2013;369(17):1579-1581

15 Maslove DM, Lamontagne F, Marshall JC, Heyland DK. A path to precision in the ICU. Crit Care 2017;21(1):79-88 